\title{
A DUALITY THEOREM FOR SOLUTIONS OF ELLIPTIC EQUATIONS PIERRE BLANCHET
}

\author{
Department of Mathematics \\ Indiana University \\ Bloomington, Indiana 47405, USA
}

(Received April 11, 1989)

\begin{abstract}
Let $\mathrm{L}$ be a second order linear partial differential operator of elliptic type on a domain $\Omega$ of $\mathbb{R}^{\mathrm{m}}$ with coefficients in $C^{\infty}(\Omega)$. We consider the linear space of all solutions of the equation $\mathrm{Lu}=0$ on $\Omega$ with the topology of uniform convergence on compact subsets and describe the topological dual of this space. It turns out that this dual may be identified with the space of solutions of an adjoint equation "near the boundary" modulo the solutions of this adjoint equation on the entire domain.
\end{abstract}

KEY WORDS AND PHRASES. Partial Differential Equations, Ellipticity, Duality. 1980 AMS SUBJECT CLASSIFICATION CODE. 35J15.

\section{INTRODUCTION AND PRELIMINARY NOTIONS.}

Let $\mathrm{H}(\mathrm{R})$ denote the linear space of harmonic functions on a noncompact Riemann surface $R$ with the topology of uniform convergence on compact subsets and let us consider the linear space $H(R)^{\prime}$ of all continuous linear functionals on $H(R)$. Nakai and Sario [1] showed that this dual can be identified with a quotient space of harmonic functions as follows:

$$
H(R)^{\prime}=H\left(\infty_{R}\right) / H(R)
$$

where $\infty_{R}$ represents the Alexandroff ideal boundary point of $R$ and where $H\left(\infty_{R}\right)$ is the space of germs of functions "harmonic at $\omega_{R}$ ".

In this work we replace $H(R)$ by the space of solutions of a linear partial differential equation and look for a similar description of the corresponding topological dual. We now introduce these solutions.

Let $\Omega$ be a domain (open connected subset) of $\mathbb{R}^{\mathrm{m}}$ with $\mathrm{m} \geq 2$. We consider the following linear partial differential operator with real valued coefficients $a_{i j}=a_{j i}, \beta_{i}$ and $c$ in $\mathrm{C}^{\infty}(\Omega)$ 


$$
L=\sum_{i, j=1}^{m} a_{i j}(x) \frac{\partial^{2}}{\partial x_{i} \partial x_{j}}+\sum_{i=1}^{m} \beta_{i}(x) \frac{\partial}{\partial x_{i}}+c(x)
$$

We assume that $L$ is of elliptic type in $\Omega$. This means that

$$
\sum_{i, j=1}^{m} a_{i j}(x) \xi_{i} \xi_{j}>0
$$

for each $\xi=\left(\xi_{1}, \ldots, \xi_{m}\right) \in \mathbb{R}^{\mathrm{m}} \backslash\{0\}$ and for each $\mathrm{x} \in \Omega$. From (1.2) we see that for every compact subset $\mathrm{K}$ of $\Omega$, there exists a constant $\lambda=\lambda(\mathrm{K})>0$ such that

$$
\lambda \sum_{i=1}^{\mathrm{m}} \xi_{\mathrm{i}}^{2} \leq \sum_{\mathrm{i}, \mathrm{j}=1}^{\mathrm{m}} \mathrm{a}_{\mathrm{ij}}(\mathrm{x}) \xi_{\mathrm{i}} \xi_{\mathrm{j}} \leq \lambda^{-1} \sum_{\mathrm{i}=1}^{\mathrm{m}} \xi_{\mathrm{i}}^{2}
$$

for each $\xi \in \mathbb{R}^{\mathrm{m}}$ and for each $\mathrm{x} \in \mathrm{K}$.

WEYL'S LEMMA. [2, page 271]. Let $f \in C^{\infty}(\Omega)$. If $u \in C^{0}(\Omega)$ satisfies,

$$
\int_{\Omega} \mathrm{u}(\mathrm{x}) \mathrm{L} \varphi(\mathrm{x}) \mathrm{d} \mathrm{x}=\int_{\Omega} \mathrm{f}(\mathrm{x}) \varphi(\mathrm{x}) \mathrm{dx}
$$

for every $\varphi \in \mathrm{C}_{\mathrm{c}}^{\infty}(\Omega)$, then $\mathrm{u} \in \mathrm{C}^{\Phi}(\Omega)$.

Therefore if $u \in C^{0}(\Omega)$ satisfies (1.4) for every $\varphi \in C_{c}^{\infty}(\Omega)$ then, at each point $x \in \Omega, u$ satisfies the adjoint equation $\mathrm{L}^{*} \mathrm{u}(\mathrm{x})=\mathrm{f}(\mathrm{x})$ where,

$$
\begin{gathered}
\mathrm{L}^{*} \mathrm{u}(\mathrm{x})=\sum_{\mathrm{i}, \mathrm{j}=1}^{\mathrm{m}} \frac{\partial^{2}}{\partial \mathrm{x}_{\mathrm{i}} \partial \mathrm{x}_{\mathrm{j}}}\left(\mathrm{a}_{\mathrm{ij}}(\mathrm{x}) \mathrm{u}(\mathrm{x})\right)- \\
\sum_{\mathrm{i}=1}^{\mathrm{m}} \frac{\partial}{\partial \mathrm{x}_{\mathrm{i}}}\left(\beta_{\mathrm{i}}(\mathrm{x}) \mathrm{u}(\mathrm{x})\right)+\mathrm{c}(\mathrm{x}) \mathrm{u}(\mathrm{x}) .
\end{gathered}
$$

The operator $\mathrm{L}^{*}$ defined by (1.5) is called the adjoint of $\mathrm{L}$.

We denote by $\mathrm{L}(\Omega)$ the set of all solutions of $\mathrm{Lu}=0$ on $\Omega$ and by $\mathrm{L}^{*}(\Omega)$ the set of all solutions of $\mathrm{L}^{*} \mathrm{u}=0$ on $\Omega$. The set $\mathrm{L}(\Omega)$ is a locally convex linear subspace of the locally convex topological linear space $C^{0}(\Omega)$. We endow $L(\Omega)$ with the topology of uniform convergence on compact subsets of $\Omega$ and we denote by $L(\Omega)^{\prime}$ the linear space of all continuous linear functionals on $L(\Omega)$.

We wish to describe $L(\Omega)^{\prime}$. In the next sections we shall identify this dual space with a certain quotient space by using a formula which relates the two operators $L$ and $L^{*}$. In the remainder of this section we state this formula and refer the reader to Miranda [3] for additional information on this. 
Let $S$ be a $C^{1}$ hypersurface in $\Omega$. If $\vec{n}(x)=\left(n_{1}(x), \ldots, n_{m}(x)\right)$ is a continuous vector field normal to $S$, then by setting

$$
a(x)=\left[\sum_{i=1}^{m}\left[\sum_{j=1}^{m} a_{i j}(x) n_{j}(x)\right]^{2}\right]^{1 / 2} \neq 0,
$$

we define a conormal to $\mathrm{S}$ as being a vector field $\vec{\nu}(\mathrm{x})=\left(\nu_{1}(\mathrm{x}), \ldots, \nu_{\mathrm{m}}(\mathrm{x})\right)$ on $\mathrm{S}$ with continuous components,

$$
\nu_{i}(x)=\frac{1}{a(x)} \sum_{j=1}^{m} a_{i j}(x) n_{j}(x) ; i=1, \ldots m
$$

and whose direction is never, by (1.2), tangent to $S$.

The directional derivative in the direction of a conormal to $\mathrm{S}$ will be,

$$
\frac{\partial}{\partial \nu} \equiv \vec{\nu} \cdot \nabla=\frac{1}{a(x)} \sum_{i, j=1}^{m} a_{i j}(x) n_{j}(x) \frac{\partial}{\partial x_{i}}
$$

and we shall call the differential operator $\partial / \partial \nu_{\mathrm{L}}=\mathrm{a}(\mathrm{x}) \partial / \partial \nu$ a conormal derivative. When $a_{i j}=\delta_{i j}$ then $a=1$ and $\vec{\nu}=\vec{n}$.

GREEN'S IDENTITY. Let $D$ be a subdomain with compact closure in $\Omega$ and with a $C^{1}$ boundary $\partial \mathrm{D}$. If $u, v \in \mathrm{C}^{2}(\overline{\mathrm{D}})$ then,

with,

$$
\int_{D}\left\{v(x) \operatorname{Lu}(x)-u(x) L^{*} v(x)\right\} d x=\int_{\partial D}[v(x), u(x)],
$$

$$
\begin{gathered}
{[v(x), u(x)]=} \\
\left\{v(x) \frac{\partial u(x)}{\partial \nu_{L}}-u(x) \frac{\partial v(x)}{\partial \nu_{L}}+e(x) u(x) v(x)\right\} d s
\end{gathered}
$$

where,

$$
\begin{gathered}
e(x)=\sum_{i=1}^{m} e_{i}(x) n_{i}(x), \\
e_{i}(x)=\beta_{i}(x)-\sum_{j=1}^{m} \partial a_{i j}(x) / \partial x_{j}, i=1, \ldots, m
\end{gathered}
$$

and where $n_{i}(x)$ are the components of the unit normal exterior to $D$. 
If $\mathrm{u}$ or $\mathrm{v}$ in (1.6) has compact support then we obtain a formula which justifies the terminology used for $\mathrm{L}^{*}$.

\section{SPACES OF GERMS AND STATEMENT OF THE DUALITY THEOREM.}

In order to describe our dual space $L(\Omega)^{\prime}$ we need to introduce now some terminology and to make some remarks.

We shall say that a subdomain $D$ of $\Omega$, relatively compact and having a $C^{\infty}$ boundary, is a normal subdomain when $\Omega \backslash \mathrm{D}$ does not have any compact connected component. An open subset $\mathrm{U}$ of $\Omega$ will be called a (punctured) neighborhood of infinity if there exists a normal subdomain $D$ of $\Omega$ such that $\Omega \backslash \bar{D} \subset U$. If $U$ is a neighborhood of infinity and if $u \in L^{*}(U)$, then we say that $(\mathrm{u}, \mathrm{U})$ (or simply $\mathrm{u}$ ) is a solution at infinity of $\mathrm{L}^{*} \mathrm{u}=0$.

Let $\mathrm{U}_{1}$ and $\mathrm{U}_{2}$ be two neighborhoods of infinity and let $\left(\mathrm{u}_{1}, \mathrm{U}_{1}\right)$ and $\left(\mathrm{u}_{2}, \mathrm{U}_{2}\right)$ be two solutions at infinity of $\mathrm{L}^{*} \mathrm{u}=0$. Then we say that $\mathrm{u}_{1}$ and $\mathrm{u}_{2}$ are equivalent if there exists a neighborhood of infinity in $\mathrm{U}_{1} \cap \mathrm{U}_{2}$ on which $\mathrm{u}_{1}$ and $\mathrm{u}_{2}$ coincide. The equivalence classes so obtained are called germs of solutions at infinity and we denote by $\mathrm{L}^{*}(\boldsymbol{w})$ the linear space of germs of solutions at infinity of $L^{*} u=0$. Here the symbol $\omega^{\infty}=\infty_{\Omega}$ represents the point such that $\Omega \cup\{\infty\}$ is the Alexandroff compactification of $\Omega$. Therefore a germ $[u]$ belongs to $\mathrm{L}^{*}(\infty)$ if $\mathrm{L}^{*} \mathrm{u}=0$ on a (punctured) neighborhood of $\infty$ in $\Omega$.

Uniqueness properties for solutions of elliptic equations were studied in a large number of works and one can obtain several references about this in Miranda [3]. The statement that follows comes from Agmon [4, page 151].

UNIQUENESS THEOREM. Let $\mathrm{L}$ be a second order linear partial differential operator of elliptic type on a domain $\Omega$ of $\mathbb{R}^{\mathrm{m}}$ with coefficients in $C^{\infty}(\Omega)$. If a $C^{2}$ solution $u$ of $L u=0$ has a zero of infinite order at a point in $\Omega$ then $u \equiv 0$.

In view of the uniqueness theorem we may identify a solution $u \in L^{*}(\Omega)$ with the germ $[u] \in L^{*}(w)$. The mapping from $L^{*}(\Omega)$ to $L^{*}(\omega)$ which maps each $u$ to its equivalence class [u] is indeed injective because if $\left[u_{1}\right]=\left[u_{2}\right]$ with $u_{1}$ and $u_{2}$ in $L^{*}(\Omega)$ then $u_{1}-u_{2}$. vanishes on a neighborhood of infinity and, therefore, identically on $\Omega$ by the uniqueness theorem.

Let $\mathrm{W}_{1}$ and $\mathrm{W}_{2}$ be two normal subdomains of $\Omega$ and let $\mathrm{u}$ and $\mathrm{v}$ be two functions of class $\mathrm{C}^{2}$ satisfying $\mathrm{Lu}=0$ and $\mathrm{L}^{*} \mathrm{v}=0$ on a neighborhood of $\Omega \backslash \mathrm{W}_{\mathrm{j}}, \mathrm{j}=1,2$. Then by applying Green's identity (1.6) on $\mathrm{W}_{0} \mid \bar{W}_{j}$ where $\mathrm{W}_{0}$ is a normal subdomain such that $\overline{\mathrm{W}}_{1} \cup \overline{\mathrm{W}}_{2} \subset \mathrm{W}_{0}$ we obtain, for $\mathrm{j}=1,2$,

$$
\begin{gathered}
\int_{\partial \mathrm{W}_{0}}[\mathrm{v}(\mathrm{x}), \mathrm{u}(\mathrm{x})]-\int_{\partial \mathrm{W}_{j}}[\mathrm{v}(\mathrm{x}), \mathrm{u}(\mathrm{x})]= \\
\mathrm{W}_{0}\left\{\overline{\mathrm{W}}_{\mathrm{j}}\left\{\mathrm{v}(\mathrm{x}) \mathrm{Lu}(\mathrm{x})-\mathrm{u}(\mathrm{x}) \mathrm{L}^{*} \mathrm{v}(\mathrm{x})\right\} \mathrm{dx}=0 .\right.
\end{gathered}
$$


Formula (2.1) means that the integral $\int_{\partial \mathrm{W}}[\mathrm{v}, \mathrm{u}]$ does not depend on the choice of the normal subdomain $W$ such that $u \in L(\Omega \backslash W)$ and $v \in L^{*}(\Omega \backslash W)$. We can therefore define the integral on the ideal boundary of $\Omega$ (obtained by compactifying $\Omega$ in the sense of Alexandroff) as follows,

$$
\int_{\infty}[v, u]=\lim _{w \rightarrow \Omega} \int_{\partial W}[v, u]
$$

whenever $u$ and $v$ are solutions at infinity of $L u=0$ and $L^{*} v=0$.

Finally, by choosing an equivalence class $\theta$ in $L^{*}(\infty)$ and an element $v \in \theta$ we see that the continuous linear functional defined by,

$$
\mathrm{L}(\Omega) \ni \mathrm{u} \longrightarrow \int_{\infty}[\mathrm{v}, \mathrm{u}]
$$

does not depend on the choice of $\mathrm{v} \in \boldsymbol{\theta}$.

We can now state our result.

DUALITY THEOREM. There is a vector-space isomorphism

$$
\mathrm{L}(\Omega)^{\prime}=\mathrm{L}^{*}(\mathrm{\Phi}) / \mathrm{L}^{*}(\Omega)
$$

where the action of a germ in $L^{*}(\infty)$ on a solution in $L(\Omega)$ is given by

$$
(\mathrm{v}, \mathrm{u})=\int_{\infty}[\mathrm{v}, \mathrm{u}]
$$

A representation similar to (2.5) was obtained by M . Nakai, L. Sario [1] for harmonic functionals on noncompact Riemann surfaces. When the operator $L$ is self adjoint, as it is in the harmonic case, then the isomorphism (2.4) reduces to the one of Nakai, Sario.

\section{SOME TOOLS.}

In order to make easier the reading of the proof of the duality theorem, we present in this section, some of the results that will be used in the next sections.

The first result is an extension, to solutions of second order linear partial differential equations of elliptic type, of the classical Runge approximation property for harmonic functions. The statement that follows comes from Lax [5, page 760].

LAX'S EQUIVALENCE THEOREM. Let $\mathrm{L}$ be a second order linear partial differential operator of elliptic type with $C^{\infty}$ coefficients in a domain $\Omega$ of $\mathbb{R}^{\mathrm{m}}$ and let $\mathrm{D}$ be a normal subdomain of $\Omega$. Every $C^{2}$ solution of $L u=0$ in $D$ is the uniform limit on compact subsets of $\mathrm{D}$ of a sequence of solutions of $\mathrm{Lu}=0$ in $\Omega$ if and only if every solution of the adjoint equation $\mathrm{L}^{*} \mathrm{u}=0$ vanishing on a hypersurface $\mathrm{S}$ and whose conormal derivative vanishes on $\mathrm{S}$ is identically zero. 
The existence and the properties of fundamental solutions for elliptic operators will also be used. We now recall the main facts connected with them and, as in section 1 , we refer the reader to Miranda [3] for additional information on the remainder of this section.

Let $\left(a_{i j}\right)$ be the matrix of coefficients of the principal part of $L$. We denote by $A_{i j}$ the elements of the inverse matrix of $\left(a_{i j}\right)$ and by $A$ the determinant of $\left(a_{i j}\right)$. We set,

$$
H(x, y)=\left\{\begin{array}{l}
\frac{1}{(m-2) \omega_{m} \sqrt{A(y)}}\left[\sum_{i, j=1}^{m} A_{i j}(y)\left(x_{i}-y_{i}\right)\left(x_{j}-y_{j}\right)\right]^{(2-m) / 2} \\
\text { for } m>2, \\
\frac{1}{2 \pi \sqrt{A(y)}} \log \left[\sum_{i, j=1}^{m} A_{i j}(y)\left(x_{i}-y_{i}\right)\left(x_{j}-y_{j}\right)\right]^{-1 / 2} \\
\text { for } m=2 .
\end{array}\right.
$$

If $r=|x-y|$ then in every relatively compact subdomain of $\Omega$ we have by (1.3),

$$
\mathrm{H}=\mathrm{O}\left(\mathrm{r}^{2-\mathrm{m}}\right), \frac{\partial \mathrm{H}}{\partial \mathrm{x}_{\mathrm{i}}}=\mathrm{O}\left(\mathrm{r}^{1-\mathrm{m}}\right), \frac{\partial^{2} \mathrm{H}}{\partial \mathrm{x}_{\mathrm{i}} \partial \mathrm{x}_{\mathrm{j}}}=\mathrm{O}\left(\mathrm{r}^{-\mathrm{m}}\right) \text {. }
$$

A function $\mathrm{E}(\mathrm{x}, \mathrm{y})$ will be called a fundamental solution of the equation $\mathrm{Lu}=0\left(\mathrm{~L}^{*} \mathrm{u}=0\right)$ on a domain $\mathrm{W}$ if it satisfies the following properties:

i) $E(x, y)$ and all its partial derivatives of first and second order with respect to the coordinates $x_{i}$ of the variable $x$ are continuous on $W \times W$ outside the diagonal.

ii) $E(x, y)$ and all its partial derivatives of first and second order with respect to the coordinates $x_{i}$ of the variable $x$ satisfy, for some $\lambda>0$, the following bounds,

$$
\begin{gathered}
\mathrm{E}-\mathrm{H}=\mathrm{O}\left(\mathrm{r}^{\lambda+2-\mathrm{m}}\right), \frac{\partial(\mathrm{E}-\mathrm{H})}{\partial \mathrm{x}_{\mathrm{i}}}=\mathrm{O}\left(\mathrm{r}^{\lambda+1-\mathrm{m}}\right), \\
\frac{\partial^{2}(\mathrm{E}-\mathrm{H})}{\partial \mathrm{x}_{\mathrm{i}} \partial \mathrm{x}_{\mathrm{j}}}=\mathrm{O}\left(\mathrm{r}^{\lambda-\mathrm{m}}\right),
\end{gathered}
$$

uniformly on every relatively compact subdomain of $\mathrm{W}$.

iii) $L_{x} E(x, y)=0\left(L_{x}^{*} E(x, y)=0\right)$ for each $x \in W$ with $x \neq y$.

Any fundamental solution $\mathrm{E}(\mathrm{x}, \mathrm{y})$ on a domain $\mathrm{W}$ together with all its partial derivatives of first order with respect to the coordinates $x_{i}$, are locally integrable in each variable and also on 
$\mathrm{W} \times \mathrm{W}$. Moreover if $\mathrm{E}(\mathrm{x}, \mathrm{y})$ is a fundamental solution of the equation $\mathrm{L}^{*} \mathrm{u}=0$ then by $[3$, page 19, (9.3)] we have $L_{x}^{*} E(x, y)=\delta_{y}$. Therefore $E$ is a fundamental solution in the sense of distribution theory.

GIRAUD'S THEOREM. [3, page 66]. Let $\Omega$ be a domain in $\mathbb{R}^{\mathrm{m}}$ and let $\mathrm{y}$ be a point of $\Omega$. Each $C^{2}$ solution $u$ of $L u=0$ in $\Omega \backslash\{y\}$ satisfying,

$$
u(x)=o(H(x, y)) \text { when } x \rightarrow y,
$$

extends to a solution of $\mathrm{Lu}=0$ on the entire domain $\Omega$.

\section{PROOF OF FORMULA 2.5.}

We set,

$$
F(\theta)(u)=\int_{\infty}[v, u],
$$

and obtain a linear mapping,

$$
\mathbf{F}: \mathbf{L}^{*}(\boldsymbol{\infty}) \quad \longrightarrow \quad \mathrm{L}(\Omega)^{\prime}
$$

Given $\ell \in L(\Omega)^{\prime}$, we look for $\theta \in L^{*}(\infty)$ such that $F(\theta)=\ell$. We first extend $\ell$ to a continuous linear functional on $C^{0}(\Omega),[6$, page 108]. Then, [1, page 154], there exists a signed regular Borel measure $\mu$ with compact support in $\Omega$ such that,

$$
\ell(u)=\int \mathrm{ud} \mu
$$

for every $u \in L(\Omega)$.

We denote by $\mathrm{S}_{\mu}$ the intersection of all normal subdomains of $\Omega$ containing the support of $\mu$. Then $\mathrm{S}_{\mu}$ is a compact subset of $\Omega$ and contains the support of $\mu$. Let $\left\{\mathrm{W}_{\mathrm{j}}\right\}_{\mathrm{j}=1}^{\infty}$ be an exhaustion of $\Omega$ by normal subdomains such that $S_{\mu} \subset W_{1}$. For each integer $j \geq 1$, let $E_{j}(x, y)$ be a fundamental solution of the equation $L^{*} v=0$ on $W_{j}$. Since $L^{*}$ has its coefficients in $C^{\infty}(\Omega)$ and since $W_{j}$ has compact closure in $\Omega$, the existence of $E_{j}(x, y)$ is guaranteed, [3, page $64]$.

For each $j \geq 1$, we define a continuous function $v_{j}(x)$ by setting,

$$
\mathbf{v}_{\mathrm{j}}(\mathrm{x})=\int \mathrm{E}_{\mathrm{j}}(\mathrm{x}, \mathrm{y}) \mathrm{d} \mu(\mathrm{y})
$$

for every $x \in \mathrm{W}_{\mathrm{j}} \backslash \mathrm{S}_{\mu}$ with $\mu$ given by (4.2).

Let $\varphi \in C_{c}^{\infty}\left(W_{j} \backslash S_{\mu}\right)$. Since $E_{j}(x, y)$ is integrable, we find by using Fubini's theorem and 
Green's identity on a regular domain $G$ such that $\operatorname{supp} \varphi c G \subset \mathrm{W}_{\mathrm{j}} \mid \mathrm{S}_{\mu}$,

$$
\mathrm{W}_{\mathrm{j}} \int_{S_{\mu}} \mathrm{v}_{\mathrm{j}}(\mathrm{x}) \mathrm{L} \varphi(\mathbf{x}) \mathrm{d} \mathbf{x}=0
$$

and we conclude by Weyl's lemma that $\mathrm{v}_{\mathrm{j}} \in \mathrm{C}^{\infty}\left(\mathrm{W}_{\mathrm{j}} \mid \mathrm{S}_{\mu}\right)$ and that $\mathrm{L}^{*} \mathrm{v}_{\mathrm{j}}(\mathrm{x})=0$ on $\mathrm{W}_{\mathrm{j}} \backslash \mathrm{S}_{\mu}$.

Let $\mathrm{D}$ be a normal subdomain such that $\mathrm{S}_{\mu} \subset \mathrm{D} \subset \overline{\mathrm{D}} \subset \mathrm{W}_{1}$. If $\mathrm{u} \in \mathrm{L}(\Omega)$ then by Green's identity we have,

$$
\int_{\partial D}\left[\mathrm{v}_{\mathrm{j}+1}(\mathrm{x}), \mathrm{u}(\mathrm{x})\right]=\int_{\partial \mathrm{W}_{\mathrm{j}}}\left[\mathrm{v}_{\mathrm{j}+1}(\mathrm{x}), \mathrm{u}(\mathrm{x})\right]
$$

for each $\mathrm{j} \geq 1$ and,

$$
\mathrm{u}(\mathrm{y})=\int_{\partial \mathrm{W}_{\mathrm{j}}}\left[\mathrm{E}_{\mathrm{j}+1}(\mathrm{x}, \mathrm{y}), \mathrm{u}(\mathrm{x})\right],
$$

for each $y \in \mathrm{W}_{\mathrm{j}}$, [3, page 19]. Therefore by Fubini's theorem and by formulas (4.3), (4.4), (4.5) and (4.2) we find,

$$
\begin{aligned}
& \int_{\partial D}\left[v_{j+1}(x), u(x)\right]=\int_{\partial W_{j}}\left[\int E_{j+1}(x, y) d \mu(y), u(x)\right]= \\
& \iint_{\partial W_{j}}\left[E_{j+1}(x, y), u(x)\right] d \mu(y)=\int u(y) d \mu(y)=\ell(u),
\end{aligned}
$$

and from this we conclude that,

$$
\ell(\mathrm{u})=\int_{\partial \mathrm{D}}\left[\mathrm{v}_{\mathrm{j}}(\mathrm{x}), \mathrm{u}(\mathrm{x})\right]
$$

for each $u \in L(\Omega)$ and each $j \geq 2$.

In order to complete the proof we wish to replace $\mathrm{v}_{\mathrm{j}}$ in (4.6) by an element $\mathrm{v} \in \mathrm{L}^{*}\left(\Omega \backslash \mathrm{S}_{\mu}\right)$.

We fix a positive integer $t$ and consider for each positive integer $k$, functions defined by $\mathrm{v}_{\mathrm{k}}(\mathrm{x})=\mathrm{v}_{\mathrm{t}+\mathrm{k}+1}(\mathrm{x})-\mathrm{v}_{\mathrm{t}+\mathrm{k}}(\mathrm{x})$ on $\mathrm{w}_{\mathrm{t}+\mathrm{k}} \mid \mathrm{S}_{\mu}$. By Giraud's theorem, the two fundamental solutions $E_{t+k+1}(x, y)$ and $E_{t+k}(x, y)$ differ at most on $W_{t+k}$ by a function $g_{k}(x, y)$ of class $C^{2}$ in the variable $x$ satisfying $L_{x}^{*} g_{k}(x, y)=0$ on $W_{t+k}$ for each $y \in W_{t+k}$. 
We claim that $g_{k}(x, y)$ is continuous on $W_{t+k} \times W_{t+k}$. To prove this we write $L^{*}$ as,

$$
L^{*}=\sum_{i, j=1}^{m} a_{i j}(x) \frac{\partial^{2}}{\partial x_{i} \partial x_{j}}+\sum_{i=1}^{m} b_{i}(x) \frac{\partial}{\partial x_{i}}+b(x)
$$

where $b_{i}(x)$ and $b(x)$ are of class $C^{\infty}$. Since $g_{k}(x, y)$ is continuous on $W_{t+k} \times W_{t+k}$ when $x \neq y$, it suffices to prove the continuity of $g_{k}(x, y)$ at those points on the diagonal.

We show that $g_{k}(x, y)$ is continuous on $B \times B$ where $B$ is any small ball in $w_{t+k}$. Let us consider such a ball $B$ sufficiently small to guaranty the existence, in a neighborhood $\omega$ of $\mathrm{B}$, of a positive $\mathrm{C}^{2}$ solution $\mathrm{w}(\mathrm{x})$ of $\mathrm{L}^{*} \mathrm{w}(\mathrm{x})=-1,[3$, page 65 and 66$]$.

For each $x \in \omega$ and each $y \in W_{t+k}$ we set,

$$
g_{k}(x, y)=\frac{g_{k}(x, y)}{w(x)} w(x) \equiv g_{k}^{1}(x, y) w(x)
$$

and we show that $g_{k}^{1}(x, y)$ is continuous on $B \times B$. Let us remark that $g_{k}^{1}(x, y)$ satisfies an elliptic equation $\mathrm{A}_{\mathrm{x}} \mathrm{g}_{\mathrm{k}}^{1}(\mathrm{x}, \mathrm{y})=0$ on $\omega$ where

$$
\begin{gathered}
A=\sum_{i, j=1}^{m} a_{i j}(x) \frac{\partial^{2}}{\partial x_{i} \partial x_{j}}+ \\
\sum_{i=1}^{m}\left[b_{i}(x)+\frac{2}{w(x)} \sum_{j=1}^{m} a_{i j}(x) \frac{\partial w(x)}{\partial x_{j}}\right] \frac{\partial}{\partial x_{i}}-\frac{1}{w(x)} .
\end{gathered}
$$

We choose a point $\left(\mathrm{x}_{1}, \mathrm{y}_{1}\right)$ in $\mathrm{B} \times \mathrm{B}$ and write,

$$
\begin{gathered}
\left|g_{k}^{1}(x, y)-g_{k}^{1}\left(x_{1}, y_{1}\right)\right| \leq\left|g_{k}^{1}(x, y)-g_{k}^{1}\left(x, y_{1}\right)\right|+ \\
\left|g_{k}^{1}\left(x, y_{1}\right)-g_{k}^{1}\left(x_{1}, y_{1}\right)\right|
\end{gathered}
$$

By the continuity of $g_{k}^{1}\left(x, y_{1}\right)$ at the point $x_{1}$, we see that the second term in the right member of inequality (4.7) can be made arbitrarily small when $x$ is close to $x_{1}$. By the maximum principle [3, page 7$]$,

$$
\left|g_{k}^{1}(x, y)-g_{k}^{1}\left(x, y_{1}\right)\right| \leq \sup _{\partial B}\left|g_{k}^{1}(\cdot, y)-g_{k}^{1}\left(\cdot, y_{1}\right)\right|
$$

for each $x$ and $y$ in $B$. But by the continuity of $g_{k}^{1}(x, y)-g_{k}^{1}\left(x, y_{1}\right)$ outside the diagonal, the right member of (4.8) and therefore those of (4.7) can be made arbitrarily small when $y$ 
approaches $\mathrm{y}_{1}$ and $(\mathrm{x}, \mathrm{y})$ approaches $\left(\mathrm{x}_{1}, \mathrm{y}_{1}\right)$. This proves the continuity of $\mathrm{g}_{\mathbf{k}}^{1}(\mathrm{x}, \mathrm{y})$ at $\left(\mathrm{x}_{1}, \mathrm{y}_{1}\right)$ and therefore in $\mathrm{B} \times \mathrm{B}$. Thus $\mathrm{g}_{\mathrm{k}}(\mathrm{x}, \mathrm{y})$ is continuous on $\mathrm{B} \times \mathrm{B}$ and therefore on $\mathrm{W}_{t+k} \times \mathrm{W}_{t+k}$. This proves the claim.

Using the claim, we extend continuously $V_{k}(x)$ by setting,

$$
\mathrm{V}_{\mathbf{k}}(\mathrm{x})=\int \mathrm{g}_{\mathbf{k}}(\mathrm{x}, \mathrm{y}) \mathrm{d} \mu(\mathrm{y})
$$

for each $x \in W_{t+k}$. Using Fubini's theorem and Green's identity as above we obtain,

$$
\int_{W_{t+k}} V_{k}(x) L \varphi(x) d x=0,
$$

for every $\varphi \in \mathrm{C}_{\mathrm{c}}^{\infty}\left(\mathrm{W}_{\mathrm{t}+\mathrm{k}}\right)$ and we conclude by Weyl's lemma that $\mathrm{V}_{\mathrm{k}} \in \mathrm{L}^{*}\left(\mathrm{~W}_{\mathrm{t}+\mathrm{k}}\right)$.

By the uniqueness theorem we may apply Lax's equivalence theorem in order to obtain, for each positive integer $k$, a global solution $h_{k} \in L^{*}(\Omega)$ such that,

$$
\sup \left\{\left|\mathrm{V}_{\mathrm{k}}(\mathrm{x})-\mathrm{h}_{\mathrm{k}}(\mathrm{x})\right|: \mathrm{x} \in \overline{\mathrm{W}}_{\mathrm{t}+\mathrm{k}-1}\right\}<1 / 2^{\mathrm{k}}
$$

Estimate (4.9) shows that the series $\sum_{k=1}^{\infty}\left(V_{k}-h_{k}\right)$ converges uniformly on compact subsets of $\Omega$.

Let us consider the series defined on $\mathrm{W}_{t+1} \backslash \mathrm{S}_{\mu}$ by,

$$
v=v_{t+1}+\sum_{k=1}^{\infty}\left(v_{k}-h_{k}\right)
$$

and whose sum belongs to $L^{*}\left(\mathrm{~W}_{\mathrm{t}+1} \backslash \mathrm{S}_{\mu}\right)$. Since $\mathrm{v}_{\mathrm{t}+\ell}+\mathrm{v}_{\ell}=\mathrm{v}_{\mathrm{t}+\ell+1}$ on $\mathrm{w}_{\mathrm{t}+\ell} \backslash \mathrm{S}_{\mu}$ for each $\ell=1, \ldots, \mathbf{k}-1$ and each $\mathbf{k} \geq 2$, we see that

$$
v_{t+1}+\sum_{i=1}^{k-1} v_{i}=v_{t+k}
$$

on $\mathrm{W}_{\mathrm{t}+1} \backslash \mathrm{S}_{\mu}$. This shows that, for each positive integer $\mathrm{k}$, the function defined on $\mathrm{W}_{\mathrm{t}+\mathrm{k}} \backslash \mathrm{S}_{\mu}$ by the series

$$
\mathrm{v}_{\mathrm{t}+\mathrm{k}}-\left(\mathrm{h}_{1}+\ldots+\mathrm{h}_{\mathrm{k}-1}\right)+\sum_{\ell=\mathrm{k}}^{\infty}\left(\mathrm{v}_{\ell}-\mathrm{h}_{\ell}\right)
$$


extends $v$ to an element of $L^{*}\left(W_{t+k} \backslash S_{\mu}\right)$. Such an extension being possible for every $k$, we see that $v$ extends to an element, still denoted by $v$, of $L^{*}\left(\Omega \backslash S_{\mu}\right)$.

Thus by applying Green's identity on a subdomain $D$ such that $S_{\mu} \subset D \subset \bar{D} \subset W_{1}$ to an element $u \in L(\Omega)$ and by using (4.6) we have,

$$
\begin{gathered}
\int_{\partial \mathrm{D}}[\mathrm{v}(\mathrm{x}), \mathrm{u}(\mathrm{x})]= \\
\int_{\partial \mathrm{D}}\left[\mathrm{v}_{\mathrm{t}+1}(\mathrm{x}), \mathrm{u}(\mathrm{x})\right]+\int_{\partial \mathrm{D} \mathbf{k}=1}\left[\sum_{\mathrm{k}}^{\infty}\left(\mathrm{V}_{\mathrm{k}}(\mathrm{x})-\mathrm{h}_{\mathbf{k}}(\mathrm{x})\right), \mathrm{u}(\mathrm{x})\right]= \\
=\ell(\mathrm{u})+\int_{\mathrm{D}}\left\{\sum_{\mathrm{k}=1}^{\infty}\left(\mathrm{V}_{\mathbf{k}}(\mathrm{x})-\mathrm{h}_{\mathrm{k}}(\mathrm{x})\right) \mathrm{Lu}(\mathrm{x})-\mathrm{u}(\mathrm{x}) \mathrm{L}^{*} \sum_{\mathrm{k}=1}^{\infty}\left(\mathrm{V}_{\mathrm{k}}(\mathrm{x})-\mathrm{h}_{\mathrm{k}}(\mathrm{x})\right)\right\} \mathrm{dx} .
\end{gathered}
$$

Therefore,

$$
\ell(\mathrm{u})=\int_{\partial \mathrm{D}}[\mathrm{v}(\mathrm{x}), \mathrm{u}(\mathrm{x})]
$$

for every $u \in L(\Omega)$. Since $v \in L^{*}\left(\Omega \backslash S_{\mu}\right)$ we obtain by (2.1) the desired representation,

$$
\ell(\mathrm{u})=\int_{\infty}[\mathrm{v}(\mathrm{x}), \mathrm{u}(\mathrm{x})],
$$

and the existence of an element $\theta=[\mathrm{v}] \in \mathrm{L}^{*}(\boldsymbol{\omega})$ such that $\mathrm{F}(\theta)=\ell$.

\section{PROOF OF ISOMORPHISM 2.4 .}

Let us denote by ker $F$ the kernel of the mapping (4.1). In order to complete the proof of the duality theorem, we have to show that ker $\mathrm{F}=\mathrm{L}^{*}(\Omega)$.

It is clear that ker $F \supset L^{*}(\Omega)$. Indeed if $v \in L^{*}(\Omega)$ then for every $u \in L(\Omega)$ we have,

$$
\begin{gathered}
F(\theta)(u)=\int_{\infty}[v, u]=\lim _{W \rightarrow \Omega} \int_{\partial W}[v, u]= \\
\lim _{w \rightarrow \Omega} \int_{W}\left\{v L u-u L^{*} v\right\} d x=0 .
\end{gathered}
$$

Conversely let us take an element $v=(v, U) \in \theta \in$ ker $F$ and a normal subdomain $W$ such that $\Omega \backslash \mathrm{W} \subset \mathrm{U}$. We extend $\mathrm{v}$ to an element $\tilde{\mathbf{v}}$ in $\mathrm{C}^{2}(\Omega)$ and we consider $\mathrm{g} \in \mathrm{C}^{2}(\mathrm{~W})$ such that $\mathrm{Lg}=0$ in $\mathrm{W}$ and $\mathrm{g}=0$ on $\partial \mathrm{W}$.

By Green's identity we have,

$$
\int_{\partial W} \mathbf{v} \frac{\partial g}{\partial \nu_{L}} d s=-\int_{W} g L^{*} \tilde{v} d \mathbf{x} .
$$


The right integral in (5.1) is taken on the support of $\mathrm{L}^{*} \tilde{\mathbf{v}}$ which is a compact subset of $\mathrm{W}$. By Lax's equivalence theorem, $\mathrm{L}(\Omega)$ is dense in $\mathrm{L}(\mathrm{W})$ and therefore there exists a sequence $\left\{g_{j}\right\}_{j=1}^{\infty}$ in $L(\Omega)$ such that $\lim _{j \rightarrow \infty} g_{j}=g$ uniformly on the support of $L^{*} \tilde{v}$. Since $v \in \theta \in$ ker $F$ we have by Green's identity,

$$
-\int_{W} g L^{*} \tilde{v} d x=-\lim _{j \rightarrow \infty} \int_{W} g_{j} L^{*} \tilde{v} d x=\lim _{j \rightarrow \infty} \int_{\partial W}\left[v, g_{j}\right]=0,
$$

and (5.1) becomes,

$$
\int_{\partial \mathrm{W}} \mathrm{v} \frac{\partial \mathrm{g}}{\partial \nu_{\mathrm{L}}} \mathrm{ds}=0
$$

Therefore, [3, pages 76, 77 and 78], (5.2) implies the existence of $h \in C^{2}(\bar{W})$ such that $L^{*} h=0$ in $\mathrm{W}$ and $\mathrm{h}=\mathrm{v}$ on $\partial \mathrm{W}$. We are going to show that there exists a function $\mathrm{h}_{1}$ satisfying these properties and such that $\partial\left(\mathrm{v}-\mathrm{h}_{1}\right) / \partial \nu_{\mathrm{L}}=0$ on $\partial \mathrm{W}$.

By Green's identity we have,

$$
\int_{W} \mathrm{uL}^{*} \tilde{\mathrm{v}} \mathrm{dx}=\int_{\partial \mathrm{W}} \mathrm{u} \frac{\partial}{\partial \nu_{\mathrm{L}}}(\mathrm{v}-\mathrm{h}) \mathrm{ds}
$$

for every $C^{2}$ solution of $\mathrm{Lu}=0$ in $\mathrm{W}$ taking boundary values of class $\mathrm{C}^{2}$ on $\partial \mathrm{W}$. By an approximation theorem of Walsh-Mergelyan type for solutions of $\mathrm{Lu}=0 \quad[7$, page 150, theorem 2], the formula (5.3) remains valid for any solutions of $\mathrm{Lu}=0$ in $\mathrm{W}$ taking continuous boundary values on $\partial \mathrm{W}$. Therefore, by Lax's equivalence theorem, formula (5.3) becomes,

$$
\int_{\partial W} \mathrm{u} \frac{\partial}{\partial \nu_{L}}(\mathrm{v}-\mathrm{h}) \mathrm{ds}=0
$$

for every $u \in L(W) \cap C^{0}(\bar{W})$.

Let us denote by $\mathrm{P}$ the prehilbert space $\mathrm{L}^{2}(\partial \mathrm{W}, \mathrm{ds}) \cap \mathrm{C}^{0}(\partial \mathrm{W})$, by $\Sigma$ the subspace of the boundary values of functions $u \in C^{0}(\bar{W})$ such that $L u=0$ in $W$ and by $\Phi$ the subspace of $P$ of all functions $\partial \varphi / \partial \nu_{\mathrm{L}}$ where $\varphi \in \mathrm{C}^{2}(\overline{\mathrm{W}})$ satisfies $\mathrm{L}^{*} \varphi=0$ in $\mathrm{W}$ and $\varphi=0$ on $\partial \mathrm{W}$. The space of such $\varphi$ has finite dimension, [3, pages 76 and 77]. Moreover if $\left\{\varphi_{\mathrm{i}}\right\}_{\mathrm{i}=1}^{\mathbf{k}}$ is a basis of this space then $\left\{\partial \varphi_{\mathrm{i}} / \partial \nu_{\mathrm{L}}\right\}_{\mathrm{i}=1}^{\mathrm{k}}$ is a finite basis of $\Phi$. Therefore, by a property of finite dimensional linear subspaces of a prehilbert space, [8, page 66, theorem 3], we have $\Phi^{\perp \perp}=\Phi$. But since we already know that $\Phi^{\perp}=\Sigma,[3$, pages 77 and 78$]$, we conclude that $\Phi=\Sigma^{\perp}$.

By (5.4), $\partial(v-h) / \partial \nu_{L} \in \Sigma^{\perp}$. Therefore, there exists $h_{0} \in C^{2}(\bar{W})$ satisfying ${ }^{2}{ }^{*} h_{0}=0$ in $\mathrm{W}, \mathrm{h}_{0}=0$ on $\partial \mathrm{W}$ and $\partial(\mathrm{v}-\mathrm{h}) / \partial \nu_{\mathrm{L}}=\partial \mathrm{h}_{0} / \partial \nu_{\mathrm{L}}$ on $\partial \mathrm{W}$. By setting $\mathrm{h}_{1}=\mathrm{h}+\mathrm{h}_{0}$, we obtain an element of $C^{2}(\bar{W})$ satisfying $L^{*} h_{1}=0$ in $W$ and $v-h_{1}=\partial\left(v-h_{1}\right) / \partial \nu L=0$ on $\partial W$. 
Let $\hat{v}$ be the function on $\Omega \backslash \partial \mathrm{W}$ which coincide with $\mathrm{v}$ on $\Omega \backslash \overline{\mathrm{W}}$ and with $\mathrm{h}_{1}$ on $\mathrm{W}$ Then by the preceding remark $\hat{\mathrm{v}}$ extends continuously to the entire domain $\Omega$ and satisfies the following relation,

$$
\int_{\Omega} \hat{\mathbf{v}}(\mathbf{x}) \mathrm{L} \varphi(\mathbf{x}) \mathrm{d} \mathbf{x}=0
$$

for every $\varphi \in \mathrm{C}_{\mathrm{c}}^{\infty}(\Omega)$. To prove (5.5) let $\varphi$ be in $\mathrm{C}_{\mathrm{c}}^{\infty}(\Omega)$ and let $\mathrm{D}$ be a domain with compact closure in $\Omega$ containing the support of $\varphi$ such that the two subdomains $D_{1}=\mathrm{D} \cap(\Omega \mid \overline{\mathrm{W}})$, $\mathrm{D}_{2}=\mathrm{D} \cap \mathrm{W}$ have a boundary of class $\mathrm{C}^{1}$. By using the fact that $\mathrm{v}-\mathrm{h}_{1}$ and its conormal derivative vanish on $\partial \mathrm{W}$, we see that the integral in (5.5) is equal to

$$
\int_{\mathrm{D}_{1}} \varphi(\mathrm{x}) \mathrm{L}^{*} \mathrm{v}(\mathrm{x}) \mathrm{d} \mathrm{x}+\int_{\mathrm{D}_{2}} \varphi(\mathrm{x}) \mathrm{L}^{*} \mathrm{~h}_{1}(\mathrm{x}) \mathrm{d} \mathrm{x}
$$

and (5.5) follows. Therefore, by Weyl's lemma $\hat{\mathrm{v}} \in \mathrm{L}^{*}(\Omega)$. Thus $[\mathrm{v}]=[\hat{\mathrm{v}}] \in \mathrm{L}^{*}(\Omega)$ and ker F C $L^{*}(\Omega)$. This proves the duality theorem.

ACKNOWLEDGMENT. The author would like to thank Paul M. Gauthier and Thomas Bagby for their interest. This work was supported by NSERC-Canada.

\section{REFERENCES}

1. Nakai, M., Sario, L. Harmonic Functionals on Open Riemann Surfaces, Pac. J. Math. 93, 1 (1981) 147-161.

2. Hörmander, L. The Analysis of Linear Partial Differential Operators I, Springer-Verlag, 1983.

3. Miranda, C. Partial Differential Equations of Elliptic Type, Second Revised Edition, Springer-Verlag, 1970.

4. Agmon, S. Unicité et Convexité dans les Problèmes Différentiels, Sém. Math. Sup. 13, Presses de l'Université de Montréal, 1966.

5. Lax, P.D. A Stability Theorem for Solutions of Abstract Differential Equations, and Its Application to the Study of the Local Behavior of Solutions of Elliptic Equations, Comm. Pure Appl. Math. 9 (1956) 747-766.

6. Yosida, K. Functional Analysis, Fifth Edition, Springer-Verlag, 1978.

7. Browder, F.E. Approximation by Solutions of Partial Differential Equations, Amer. J. Math. 84 (1962) 134-160.

8. Berberian, S.K. Introduction to Hilbert Space, Oxford University Press, 1961. 


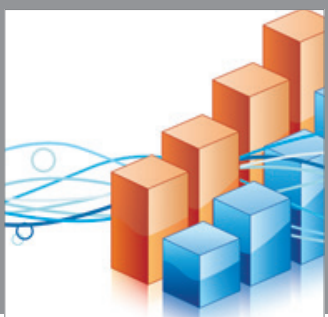

Advances in

Operations Research

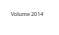

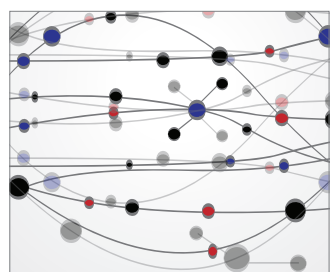

\section{The Scientific} World Journal
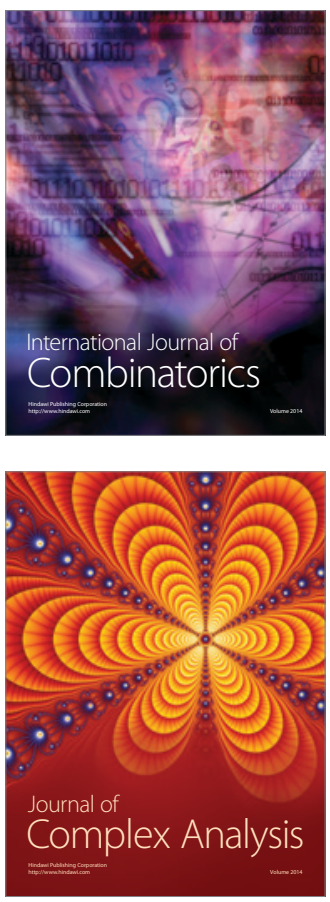

International Journal of

Mathematics and

Mathematical

Sciences
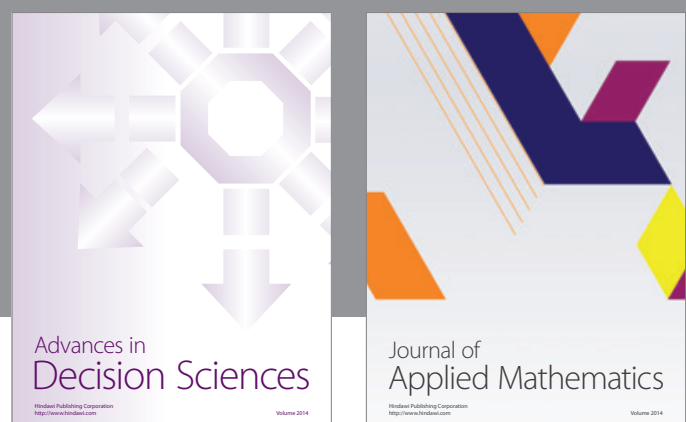

Journal of

Applied Mathematics
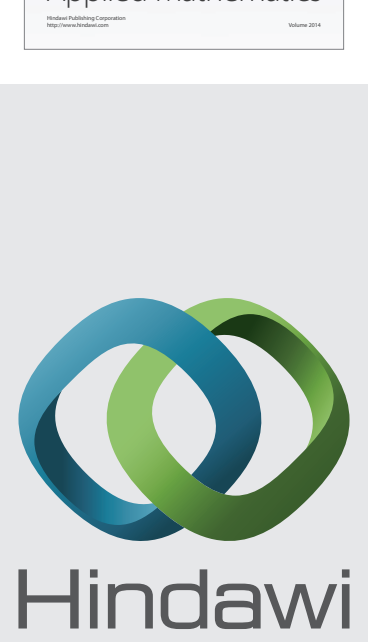

Submit your manuscripts at http://www.hindawi.com
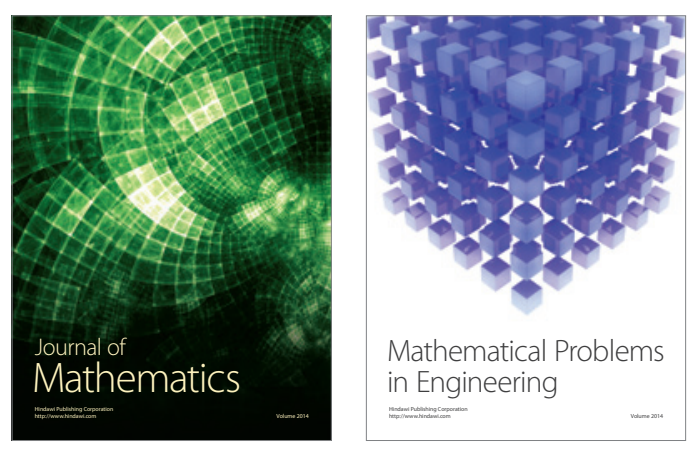

Mathematical Problems in Engineering
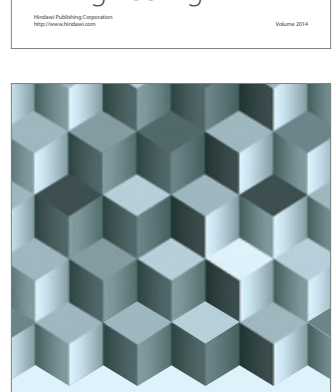

Journal of

Function Spaces
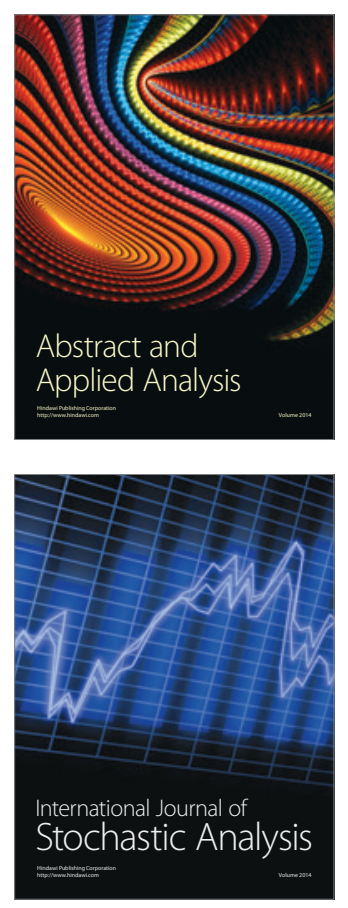

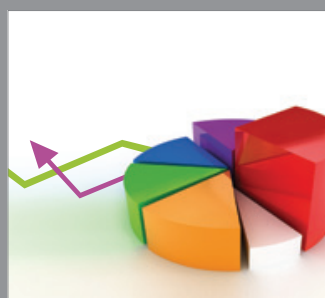

ournal of

Probability and Statistics

Promensencen
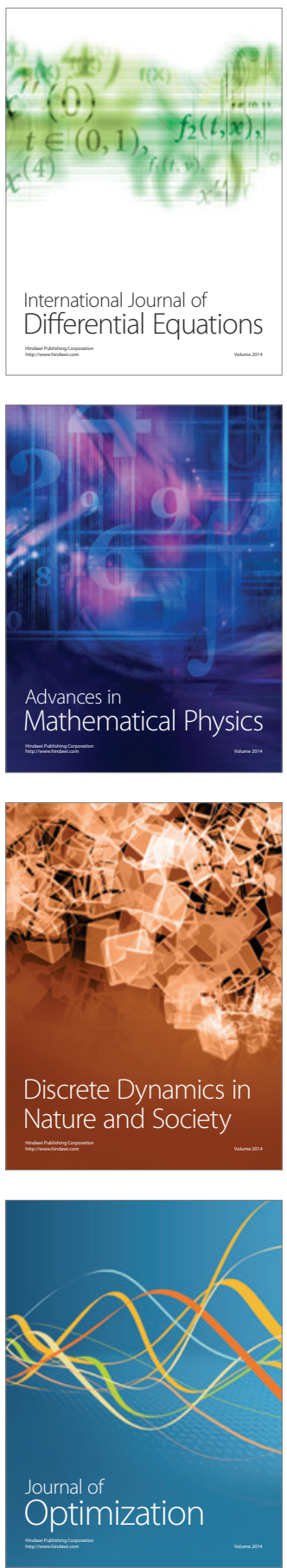